\title{
On Some Normality-Like Properties and Bishop's Property $(\beta)$ for a Class of Operators on Hilbert Spaces
}

\author{
Sid Ahmed Ould Ahmed Mahmoud \\ Mathematics Department, College of Science, Al Jouf University, Al Jouf 2014, Saudi Arabia \\ Correspondence should be addressed to Sid Ahmed Ould Ahmed Mahmoud, sidahmed@ju.edu.sa \\ Received 11 December 2011; Accepted 19 February 2012 \\ Academic Editor: Shigeru Kanemitsu \\ Copyright (C) 2012 Sid Ahmed Ould Ahmed Mahmoud. This is an open access article distributed \\ under the Creative Commons Attribution License, which permits unrestricted use, distribution, \\ and reproduction in any medium, provided the original work is properly cited. \\ We prove some further properties of the operator $T \in[n \mathrm{QN}]$ (n-power quasinormal, defined in \\ Sid Ahmed, 2011). In particular we show that the operator $T \in[n \mathrm{QN}]$ satisfying the translation \\ invariant property is normal and that the operator $T \in[n \mathrm{QN}]$ is not supercyclic provided that it is \\ not invertible. Also, we study some cases in which an operator $T \in[2 \mathrm{QN}]$ is subscalar of order $m$; \\ that is, it is similar to the restriction of a scalar operator of order $m$ to an invariant subspace.
}

\section{Introduction}

Although normality of operators $\left(T^{*} T=T T^{*}\right)$ makes things easier, it rarely occurs and relaxing the normality condition is essential in the theory of operators on Hilbert spaces. One of the most important subclasses of the algebra of all bounded linear operators acting on a Hilbert space, the class of hyponormal operators, has been studied by many authors (see [1]). In recent years this class has been generalized, in some sense, to larger classes of the socalled $p$-hyponormal, log-hyponormal, posinormal, k-quasihyponormal classes, and so forth (see $[2-6]$ ).

In [7], Putinar showed that hyponormal operators are subscalar. This fact has led to far-reaching results, discovering deep properties of these operators. In this paper we extend that result to other generalized classes of operators.

Let $H$ be an infinite dimensional separable complex Hilbert space, let $K$ be a complex Hilbert space, and let $\mathcal{L}(H, K)$ be the algebra of all bounded linear operators from $H$ to $K$. We write $\mathcal{L}(H)$ for $\mathcal{L}(H, H)$. If $T \in \mathcal{L}(H, K)$, we will write $N(T)$ and $R(T)$ for the null space (also referred to as the reducing subspace) and the range of $T$, respectively. The spectrum, 
the point spectrum, and the approximate point spectrum of an operator $T$ are denoted by $\sigma(T), \sigma_{p}(T), \sigma_{a p}(T)$, respectively. $T^{*}$ means the adjoint of $T$.

An operator $T \in \mathcal{L}(H)$ is

(1) hyponormal if and only if $T T^{*} \leq T^{*} T \Leftrightarrow\left\|T^{*} x\right\| \leq\|T x\|$, for all $x \in H$,

(2) posinormal if and only if $R(T) \subset R\left(T^{*}\right)$, or equivalently $T T^{*} \leq \lambda^{2} T^{*} T$ for some $\lambda>0$,

(3) $p$-hyponormal if and only if $\left(T T^{*}\right)^{p} \leq\left(T^{*} T\right)^{p}, 0<p \leq 1$,

(4) $p$-quasihyponormal if and only if $T^{*}\left[\left(T T^{*}\right)^{p}-\left(T^{*} T\right)^{p}\right] T \leq 0,0<p<1$,

(5) log-hyponormal if $T$ is invertible and $\log \left(T T^{*}\right) \leq \log \left(T^{*} T\right)$,

(6) 2-isometry if and only if $T^{* 2} T^{2}-2 T^{*} T+I=0$ (see [8]), where $I$ indicates the identity operator that is, if and only if

$$
T^{* 2} T^{2}=2 T^{*} T-I
$$

An operator $T \in \mathcal{L}(H)$ is said to be $n$-power quasinormal (abbreviated as $n \mathrm{QN}$ ), $n=1,2, \ldots$, if

$$
T^{n} T^{*} T=T^{*} T T^{n}=T^{*} T^{n+1} .
$$

If $n=1, T$ is called quasinormal. This class of operators being denoted by $[n \mathrm{QN}]$, that is,

$$
[n \mathrm{QN}]:=\left\{T \in \mathcal{L}(H): T^{n} T^{*} T-T^{*} T T^{n}=0\right\}
$$

was studied by the author [9].

$T$ is called an $m$-partial isometry if $T$ satisfies

$$
T B_{m}(T)=T \sum_{k=0}^{m}\left(\begin{array}{l}
m \\
k
\end{array}\right)(-1)^{k} T^{* m-k} T^{m-k}=0,
$$

where $B_{m}(T)$ is obtained formally from the binomial expansion of $B_{m}(T)=\left(T^{*} T-I\right)^{m}$ by understanding $\left(T^{*} T\right)^{m-k}=T^{* m-k} T^{m-k}$. The case when $m=1$ is called the partial isometries class. The class of $m$-partial isometries was defined by Saddi and Sid Ahmed [10] who proved some properties of the class. See Proposition 5.4.

This paper is divided into five sections. Section 2 deals with some preliminary facts concerning function spaces. Section 3 includes our main results. There we study some properties of $[n \mathrm{QN}]$. In particular we show that an operator $T \in[n \mathrm{QN}]$ satisfying the translation invariant property is normal, and an invertible operator $T \in[n \mathrm{QN}]$ and its inverse $T^{-1}$ have a common nontrivial invariant closed set provided that $T^{*} \in[n \mathrm{QN}]$. Also we show that some of class $[2 \mathrm{QN}]$ satisfy an analogue of the single-valued extension for $W_{2}^{m}(D, H)$ and have scalar extension. In Section 4 , we give some results about the Berberian extension. In Section 5, we shall use some properties of the approximate spectrum to obtain some results on single-valued extension (SVEP) (see Section 2 ) property for the $m$-partial isometries operators. 


\section{Spaces of Vector-Valued Functions}

We will need the following function spaces.

Let $\lambda$ be the coordinate in $\mathbb{C}$ and let $d \mu(\lambda)$ denote the planar Lebesgue measure. Let $D$ be a bounded open subset of $\mathbb{C}$. We will denote by $L^{2}(D, H)$ the Hilbert space of square measurable (or summable) functions $f: D \rightarrow H$ such that

$$
\|f\|_{2, D}=\left\{\int_{D}\|f(\lambda)\|^{2} d \mu(\lambda)\right\}^{1 / 2}<\infty
$$

Let $O(D, H)$ denote the space of $H$-valued functions analytic on $D$, that is, $\bar{\partial} f=$ $\partial f / \partial \bar{z}=0$. Equipped with the topology of uniform convergence on compact subsets of $D$, $O(D, H)$ is a Frechet space. Let

$$
A^{2}(D, \mathrm{H})=L^{2}(D, H) \cap O(D, H)
$$

denote the Bergman space for $D$ consisting of square measurable functions $f$ that are analytic on $D$.

We denote by $P$ the orthogonal projection of $L^{2}(D, H)$ onto $A^{2}(D, H)$.

Let us define now a special Sobolev type space. Let $m$ be a fixed nonnegative integer. The Sobolev space $W_{2}^{m}(D, H)$ of order $m$ of vector-valued functions with respect to $\bar{\partial}$ will be the space of those functions $f \in L^{2}(D, H)$ whose derivatives $\bar{\partial} f, \ldots, \bar{\partial}^{m} f$ in the sense of distributions also belong to $L^{2}(D, H)$, that is,

$$
W_{2}^{m}(D, H)=\left\{f \in L^{2}(D, H): \bar{\partial}^{k} f \in L^{2}(D, H), \text { for } k=0,1, \ldots, m\right\}
$$

Endowed with the norm

$$
\|f\|_{W_{2}^{m}}^{2}=\sum_{k=0}^{m}\left\|\bar{\partial}^{k} f\right\|_{2, D}^{2} .
$$

$W_{2}^{m}(D, H)$ becomes a Hilbert space contained continuously in $L^{2}(\mathrm{D}, H)$; that is, there is a constant $0<C<\infty$ such that $\|f\|_{L^{2}(D, H)} \leq C\|f\|_{W_{2}^{m}(D, H)}$ for all $f \in W_{2}^{m}(D, H)$.

Let

$$
T_{\lambda}=T-\lambda=T-\lambda I
$$

for $\lambda \in \mathbb{C}$ once and for all, whenever the definition is meaningful. We say that $T$ has the singlevalued extension property at $\lambda_{0} \in \mathbb{C}$ (abbreviated SVEP at $\lambda_{0}$ ) if, for every open neighborhood $U$ of $\lambda_{0}$, the only analytic solution $f$ to the equation

$$
\left(T_{\lambda} f\right)(\lambda)=(T-\lambda) f(\lambda)=0
$$


for all $\lambda$ in $U$ is the constant function $f \equiv 0$. We say that $T$ has SVEP if $T$ has a SVEP at every $\lambda \in \mathbb{C}$. $D \subseteq \mathbb{C}$.

It is easily seen that an operator $T \in \mathcal{L}(H)$ has SVEP if and only if, for each open

The operator $T_{D}: O(D, H) \rightarrow O(D, H)$ defined by

$$
T_{D}(f)(\lambda)=T_{\lambda} f(\lambda) \quad \forall f \in O(D, H), \lambda \in D
$$

is one to one.

Recall that, for a bounded operator $T$ on $H$, the local resolvent set $\rho_{T}(x)$ of $T$ at the point $x \in H$ is defined as the union of all open subsets $D$ of $\mathbb{C}$ such that there exists an analytic function $f: D \rightarrow H$ which satisfies

$$
\left(T_{\lambda}\right) f(\lambda)=x \quad \forall \lambda \in D
$$

The local spectrum $\sigma_{T}(x)$ of $T$ at $x \in H$ is the set defined by $\sigma_{T}(x):=\mathbb{C} \backslash \rho_{T}(x)$ and obviously $\sigma_{T}(x) \subset \sigma(T)$. It is clear from the definition that, $T$ has SVEP if and only if zero is the unique vector $x \in H$ such that $\sigma_{T}(x)=\emptyset$ (see for more details [11]).

Recall that a bounded operator $T \in \mathcal{L}(H)$ is said to have the Bishop's property $(\beta)$ if for every open subset $D$ of the complex plane $\mathbb{C}$ and every sequence of analytic functions $f_{n}: D \rightarrow H$ with the property that

$$
T_{\lambda} f_{n}(\lambda) \longrightarrow 0 \text { as } n \longrightarrow \infty,
$$

uniformly on all compact subsets of $D, f_{n}(\lambda) \rightarrow 0$ as $n \rightarrow \infty$ locally uniformly on $D$ or equivalently, for every open subset $D$ of $\mathbb{C}$, the operator $T_{D}$ defined in (2.7) is one to one and has the closed range [11, Proposition 3.3.5]. It is a very important notion in spectral theory. It is wellknown that every normal operator has Bishop's property $(\beta)$.

A bounded operator $T$ on $H$ is called scalar of order $m$ if it possesses a spectral distribution of order $m$, that is, if there is a continuous unital morphism,

$$
\Phi: C_{0}^{m}(\mathbb{C}) \longrightarrow \mathcal{L}(H)
$$

such that $\Phi(z)=T$, where $z$ stands for the identity function on $\mathbb{C}$ and $C_{0}^{m}(\mathbb{C})$ for the space of compactly supported functions on $\mathbb{C}$, continuously differentiable of order $m, 0 \leq m \leq \infty$. An operator is subscalar if it is similar to the restriction of a scalar operator to an invariant subspace.

Let $M_{z}$ be the operator on $W_{2}^{m}(D ; H)$ such that $\left(M_{z} f\right)(z)=z f(z)$ for $f \in W_{2}^{m}(D ; H)$. This has a spectral distribution of order $m$, defined by the functional calculus $\Phi_{M}: C_{0}^{m}(\mathbb{C}) \rightarrow$ $\mathcal{L}\left(W_{2}^{m}(D, H)\right) ; \Phi_{M}(f)=M_{f}$. Therefore $M_{z}$ is a scalar operator of order $m$. Consider a bounded open disk $D$ which contains $\sigma(T)$ and the quotient space

$$
H(D)=\frac{W_{2}^{m}(D, H)}{\overline{T_{z} W_{2}^{m}(D, H)}}
$$


endowed with the Hilbert space norm. We denote the class containing a vector $f$ or an operator A on $H(D)$ by $\widehat{f}$ or $\widehat{A}$, respectively. Let $M_{z}$ be the operator of multiplication by $z$ on $W_{2}^{m}(D ; H)$. As noted above, $M_{z}$ is a scalar of order $m$ and has a spectral distribution $\Phi$. Let $S \equiv \widehat{M}_{z}$. Since $\overline{T_{z} W_{2}^{m}(D, H)}$ is invariant under every operator $M_{f} ; f \in C_{0}^{m}(\mathbb{C})$, we infer that $S$ is a scalar operator of order $m$ with spectral distribution $\widehat{\Phi}$. Consider the natural map $V: H \rightarrow H(D)$ defined by $V h=[\widehat{1 \otimes h}]$, for $h \in H$, where $1 \otimes h$ denotes the constant function identically equal to $h$. In [7], Putinar showed that if $T \in \mathcal{L}(H)$ is a hyponormal operator then $V$ is one to one and has closed range such that $V T=S V$, and so $T$ is subscalar of order $m$.

\section{Further Properties of the Class $[n \mathbf{Q N}]$}

We start this section with some properties of $n$-power quasinormal operators.

Theorem 3.1. The class $[n Q N]$ has the following properties.

(1) The class $[n Q N]$ is closed under unitary equivalence and scalar multiplication.

(2) If $T$ is of class $[n Q N]$ and $M$ is a closed subspace of $H$ that reduces $T$, then $T \mid M$ (the restriction of $T$ to $M)$ is of class $[n Q N]$.

Proof. (1) Let $S \in \mathcal{L}(H)$ be unitary equivalent to $T$. Then there is a unitary operator $V \in \mathcal{L}(H)$ such that $T=V^{*} S V$ which implies that $T^{*}=V^{*} S^{*} V$. Noting that $T^{n}=V^{*} S^{n} V$ and inserting $I=V V^{*}$ suitably, we deduce from (1.2) that

$$
V^{*} S^{n} S^{*} S V=T^{n} T^{*} T=T^{*} T^{n+1}=V^{*} S^{*} S^{n+1} V,
$$

and (1.2) follows for S. Since

$$
(T \mid M)^{\Delta}=\left(T^{\Delta} \mid M\right)
$$

for $\Delta$ as the $n$-th power or the adjoint, it follows that the left-hand side of (1.2) for $(T \mid M)$ reads

$$
\left(T^{n} T^{*} T \mid M\right)
$$

which is $\left(T^{*} T^{n+1} \mid M\right)=(T \mid M)^{*}(T \mid M)^{n+1}$, which is the right-hand side of (1.2). Thus, $T \mid M$ is of class $[n \mathrm{QN}]$.

Next we characterize a matrix on a 2-dimensional complex Hilbert space which is in $[n \mathrm{QN}]$. Since every matrix on a finite dimensional complex Hilbert space is unitarily equivalent to an upper triangular matrix and an $n$-power quasinormal operator is unitarily invariant, it suffices to characterize an upper triangular matrix $T$. From the direct calculation, we get the following characterization. 
Proposition 3.2. For $n \geq 2$ one has

$$
T=\left(\begin{array}{ll}
x & y \\
0 & z
\end{array}\right) \in[n Q N] \Longleftrightarrow\left\{\begin{array}{l}
\bar{y} x\left(x^{n-1}+z x^{n-2}+\cdots+z^{n-1}\right)=0 \\
x \bar{y}\left(z^{n}-x^{n}\right)=0, \\
\bar{x} y\left(z^{n}-x^{n}\right)-\left(x^{n-1}+z x^{n-2}+\cdots+z^{n-1}\right)\left(|y|^{2}+|z|^{2}-|x|^{2}\right)=0
\end{array}\right.
$$

We remark here that Proposition 3.2 offers the convenient criterion to find some examples of operators in $[n \mathrm{QN}]$. Also we observe that $[n \mathrm{QN}]$ is not necessarily normal on a finite dimensional space.

Next couple of results show that $[n \mathrm{QN}]$ does not have a translation invariant property.

Theorem 3.3 (see [9]). If $T$ and $T-I$ are of class [2QN], then $T$ is normal.

Theorem 3.4 (see [9]). If $T$ is of class $[2 Q N] \cap[3 Q N]$ such that $T-I$ is of class $[n Q N]$, then $T$ is normal.

It is natural to ask the following question: what is the operators in [ $n \mathrm{QN}]$ satisfying the translation invariant property? The answer to this question is provided by the following theorem.

Theorem 3.5. $T_{\lambda}$ is of class $[n Q N]$ for every $\lambda \in \mathbb{C}$ if and only if $T$ is a normal operator.

Proof. Assume that $\left(T_{\lambda}\right)$ is of class $[n \mathrm{QN}]$ for every $\lambda \in \mathbb{C}$. Then (1.2) reads $(T-\lambda)^{n}(T-\lambda)^{*}(T-$ $\lambda)=(T-\lambda)^{*}(T-\lambda)(T-\lambda)^{n}$, which reduces on eliminating the common factor $-\bar{\lambda}(T-\lambda)^{n+1}$ to

$$
(T-\lambda)^{n}\left(T^{*} T-\lambda T^{*}\right)=\left(T^{*} T-\lambda T^{*}\right)(T-\lambda)^{n} .
$$

By the binomial expansion,

$$
\sum_{k=0}^{n}(-1)^{k}\left(\begin{array}{l}
n \\
k
\end{array}\right) \lambda^{k} T^{n-k}\left(T^{*} T-\lambda T^{*}\right)=\left(T^{*} T-\lambda T^{*}\right) \sum_{k=0}^{n}(-1)^{k}\left(\begin{array}{l}
n \\
k
\end{array}\right) \lambda^{k} T^{n-k},
$$

whence by arranging terms suitably, the extremal terms vanishing in view of (1.2),

$$
\sum_{k=1}^{n-1}(-1)^{k}\left(\begin{array}{l}
n \\
k
\end{array}\right) \lambda^{k}\left(T^{n-k} T^{*} T-T^{*} T T^{n-k}\right)-\sum_{k=1}^{n-1}(-1)^{k}\left(\begin{array}{l}
n \\
k
\end{array}\right) \lambda^{k+1}\left(T^{n-k} T^{*}-T^{*} T^{n-k}\right)=0 .
$$

Now note that from the second summand in (3.7), we may extract the extremal term $(-1)^{n} n \lambda^{n}\left(T^{*} T-T T^{*}\right)$ and express it in terms of the remaining terms which contain $\lambda$ to the power $<n$. Hence dividing (3.7) by $\lambda^{n}$ and letting $\lambda \rightarrow \infty$, we conclude that $T^{*} T-T T^{*} \rightarrow 0$, whence the normality of $T$ follows.

Conversely it is known that normality is a translation invariant property; that is, if $T$ is normal, then $\left(T_{\lambda}\right)$ is normal for every $\lambda \in \mathbb{C}$, and hence $\left(T_{\lambda}\right)$ is of class $[n \mathrm{QN}]$. 

operator.

The following proposition gives a characterization of an $n$-power quasinormal

Proposition 3.6. Let $T \in \mathcal{L}(H), A=T^{n}+T^{*} T$, and $B=T^{n}-T^{*} T$. Then $T$ is of class [ $\left.n Q N\right]$ if and only if $A$ commutes with $B$.

Proof. Commutativity of $A$ and $B$ is equivalent to (1.2).

Proposition 3.7. Let $T, A, B$ be as in Proposition 3.6. Then if $T$ is of class $[n Q N]$, then $T^{n} T^{*} T$ commutes with $A$ and $B$.

Proof. By (1.2),

$$
T^{n} T^{*} T\left(T^{n} \pm T^{*} T\right)=\left(T^{n} \pm T^{*} T\right) T^{n} T^{*} T
$$

In general, the two classes $[n \mathrm{QN}]$ and $[(n+1) \mathrm{QN}]$ are not the same (see [9]).

Proposition 3.8. If $T$ is both of class $[n Q N]$ and $[(n+1) Q N]$, then it is of class $[(n+2) Q N]$, that is, $[n Q N] \cap[(n+1) Q N] \subset[(n+2) Q N]$.

Proof. By (1.2),

$$
T^{n+1} T^{*} T=T^{n} T^{*} T^{2}
$$

so that $T^{n+2} T^{*} T$ may be transformed into $T^{*} T T^{n+2}$.

It is known that if $T$ belongs to $[n \mathrm{QN}]$ some $n>0, T^{2}$ does not necessarily belong to the same class.

Theorem 3.9 (see [9]). If $T$ and $T^{*}$ are of class $[n Q N]$, then $T^{n}$ is normal.

Proposition 3.10. If an operator $T$ of class $[2 Q N]$ is a 2-isometry, then $T^{2}$ is of class $[n Q N]$ for all integers $n \geq 2$.

Proof. From Proposition 3.8 it suffices to prove that $T^{2}$ is of class [2QN] and of class [3QN] because we may then proceed inductively.

Since $T$ is a 2-isometry, we have $T^{4}\left(T^{* 2} T^{2}\right)=T^{4}\left(2 T^{*} T-I\right)$. Using $T^{2} T^{*} T=T^{*} T^{3}$, we may shift the power of $T^{2}$ to the left, arriving at $T^{4}\left(T^{* 2} T^{2}\right)=T^{* 2} T^{6}$; that is, $T^{2}$ is of class [2QN].

In the same way, we may deduce that $T^{6}\left(T^{* 2} T^{2}\right)=T^{* 2} T^{8}$ whence $T^{2}$ is of class [3QN].

Lemma 3.11 (see [9]). If $T$ is of class $[n Q N]$, then $N\left(T^{n}\right) \subset N\left(T^{* n}\right)$.

Proposition 3.12. If $T$ is both of class $[n Q N]$ and $[(n+1) Q N]$ such that $T$ is injective or $T^{*}$ is injective, then $T$ is quasinormal.

Proof. Since $T$ is of class $[n \mathrm{QN}] \cap[(n+1) \mathrm{QN}]$, we have (3.9), which reads $T^{n}\left(T T^{*} T-T^{*} T^{2}\right)=0$. If $T$ is injective, then so is $T^{n}$ and we have $T T^{*} T-T^{*} T^{2}=0$, whence $T$ is quasi-normal. If $T^{*}$ is injective, we may appeal to Lemma 3.11. 
In the following theorem we prove some topological properties of the class $[n \mathrm{QN}]$.

Theorem 3.13. The class $[n Q N]$ is an arcwise-connected, closed subset of $\mathcal{L}(H)$ equipped with the uniform operator (norm) topology.

Proof. By Theorem 3.1, (1), we see that the ray $a T$ in $\mathcal{L}(H)$ through $T$ is contained in $[n \mathrm{QN}]$ for every complex number $a$, and therefore $[n \mathrm{QN}]$ is arcwise-connected.

To see that $[n \mathrm{QN}]$ is closed, we prove that any strong limit $T \in \mathcal{L}(H)$ of a sequence $\left(T_{p}\right)$ in $[n \mathrm{QN}]$ also belongs to $[n \mathrm{QN}]$; that is, we let $\left(T_{p}\right)$ be a sequence of operators in $[n \mathrm{QN}]$ converging to $T \in \mathcal{L}(H)$ in norm:

$$
\left\|T_{p} x-T x\right\| \longrightarrow 0 \quad \text { as } p \longrightarrow \infty \text {, for each } x \in H \text {. }
$$

Hence it follows that

$$
\left\|T_{p}^{*} x-T^{*} x\right\|=\left\|\left(T_{p}-T\right)^{*} x\right\| \leq\left\|\left(T_{p}-T\right)^{*}\right\|\|x\|=\left\|T_{p}-T\right\|\|x\| \longrightarrow 0,
$$

whence $\left(T_{p}^{*}\right)$ converges strongly to $T^{*}$.

Since the product of operators is sequentially continuous in the strong topology (see [12, page 62]), one concludes that $T_{p}^{n} T_{p}^{*} T_{p}$ converge strongly to $T^{n} T^{*} T$. Similarly $T_{p}^{*} T_{p}^{n+1}$ converges strongly to $T^{*} T^{n+1}$. Hence the limiting case of (1.2) shows that $T$ belongs to [ $n \mathrm{QN}$ ], completing the proof.

Proposition 3.14. If $T_{1}, T_{2}, \ldots, T_{p}$ are of class $[n Q N]$, then both the direct sum $T_{1} \oplus T_{2} \oplus \cdots \oplus T_{p}$ and the tensor product $T_{1} \otimes T_{2} \otimes \cdots \otimes T_{p}$ are of class $[n Q N]$.

Proof. By the compatibility principle similar to (3.2),

$$
\left(T_{1} \oplus T_{2}\right)^{\Delta}=\left(T_{1}^{\Delta} \oplus T_{2}^{\Delta}\right), \quad\left(T_{1} \otimes T_{2}\right)^{\Delta}=\left(T_{1}^{\Delta} \otimes T_{2}^{\Delta}\right),
$$

where $\Delta$ indicates either the $n$th power or adjoint, the proof follows.

A linear operator $T$ on $H$ is hypercyclic if there is a vector with dense orbit; that is, if there exists an $x \in H$ such that orbit $\operatorname{Orb}(T, x):=\left\{x, T x, T^{2} x, \ldots\right\}$ is dense in $H$, and in this case $x$ is called a hypercyclic vector for $T$.

An operator $T$ on $H$ is supercyclic if there exists a vector whose scaled orbit is dense; that is, if there exists an $x \in H$ such that $\left\{\lambda T^{n} x, n \geq 0, \lambda \in \mathbb{C}\right\}$ is dense in $H$ and in this case $x$ is called a supercyclic vector for $T$.

Kitai [13] showed that hyponormal operators are not hypercyclic. We generalize Kitai's theorem to the class $[n \mathrm{QN}]$.

Proposition 3.15. If $T$ is of class $[n Q N]$ with $\sigma_{p}\left(T^{n}\right) \neq \emptyset$, then $T$ is not hypercyclic.

Proof. If $T$ is hypercyclic, $T^{n}$ is hypercyclic, and hence $\sigma_{p}\left(T^{* n}\right)=\emptyset$ by [13, corollary 2.4]. From Lemma 3.11 we have $\sigma_{p}\left(T^{n}\right) \subset \sigma_{p}\left(T^{* n}\right)$ and, hence, $\sigma_{p}\left(T^{n}\right)=\emptyset$, a contradiction.

Theorem 3.16 (see [14, Theorem 2]). If $T$ is not hypercyclic, then $T$ and $T^{-1}$ have a common nontrivial invariant closed subset. 
Proposition 3.17. If $T$ is of class $[n Q N]$ and $0 \notin \sigma(T)$, then $T$ and $T^{-1}$ have a common nontrivial closed invariant subset.

Proof. Since $T$ is of class $[n \mathrm{QN}]$ and $0 \notin \sigma(T)$, it follows that $T^{n}$ is normal, and hence $\left(T^{-1}\right)^{n}$ is normal. By [13, Corollary 4.5] $T^{n}$ and $\left(T^{-1}\right)^{n}$ have no hypercyclic vector. Thus by [15], neither $T$ or $T^{-1}$ has a hypercyclic vector. Therefore by [15] $T$ and $T^{-1}$ have a common nontrivial closed invariant subset. Hence Theorem 3.16 completes the proof.

Proposition 3.18. Operators $T$ that are of class $[n Q N]$ such that $T$ is not invertible are not supercyclic.

Proof. Assume that $T$ is of class $[n \mathrm{QN}]$ and supercyclic. Considering the class $[n \mathrm{QN}]$ being closed under multiplication by nonzero scalars, we may assume that $\|T\|=1$. Since the supercyclic contraction $T$ satisfies property $(\beta), \sigma(T)$ is contained in the boundary $\partial \mathbb{D}$ of the unit disk $\mathbb{D}$ [11, Proposition 3.3.18]. Thus $T$ is invertible, and we have a contradiction.

Definition 3.19. An operator $T \in \mathcal{L}(H)$ is algebraic if there is non-zero-polynomial $p$ such that $p(T)=0$.

The following proposition shows that some quasinilpotent $n$-power quasi-normal operators are subscalar.

Proposition 3.20. If both $T$ and $T^{*}$ are of class $[n Q N]$ such that $T$ is quasinilpotent, then $T$ is nilpotent, and hence $T$ is subscalar.

Proof. Since $T$ is quasinilpotent, $\sigma(T)=\{0\}$. Hence by the spectral mapping theorem we get $\sigma\left(T^{n}\right)=\sigma(T)^{n}=\{0\}$. Thus $T^{n}$ is quasinilpotent and normal. So $T^{n}=0$; that is, $T$ is nilpotent, and $T$ is algebraic operator, and hence $T$ is subscalar.

Proposition 3.21 (see [7, Proposition 2.1]). For every bounded disk $D$ in $\mathbb{C}$, there is a constant $C_{D}$ such that for an arbitrary operator $T \in \mathcal{L}(H)$ and $f \in W_{2}^{m}(D, H)$ we have

$$
\left\|(I-P) \bar{\partial}^{j} f\right\|_{2, D} \leq C_{D}\left(\left\|\left(T_{\curlywedge}\right)^{*} \bar{\partial}^{j+1} f\right\|_{2, D}+\left\|\left(T_{\curlywedge}\right)^{*} \bar{\partial}^{j+2} f\right\|_{2, D}\right), \quad j=0,1, \ldots, m-2,
$$

where $P$ is the orthogonal projection of $L^{2}(D, H)$ onto $A^{2}(D, H)$.

The next theorem is important for the proof of our main theorem, Theorem 3.27.

Theorem 3.22. Let $D$ be an arbitrary bounded disk in $\mathbb{C}$. If $T$ is of class $[2 Q N]$ and $\sigma(T) \cap(-\sigma(T))=$ $\emptyset$, then the operator

$$
T_{\lambda}: W_{2}^{m}(D, H) \longrightarrow W_{2}^{m}(D, H)
$$

is one to one. 
Proof. Let $g \in W_{2}^{m}(D, H)$ such that $T_{\lambda} g=0$, that is,

$$
\left\|T_{\curlywedge} g\right\|_{W_{2}^{m}}=0 .
$$

Then for $j=0,1, \ldots, m$ we have

$$
\left\|T_{\lambda} \bar{\partial}^{j} g\right\|_{2, D}=0
$$

Hence for $j=1, \ldots, m$ we get $\left\|T_{\lambda^{2}}^{2} \bar{\partial}^{j} g\right\|_{2, D}=0$. Since $\sigma(T) \cap(-\sigma(T))$ is empty then $T^{2}$ is normal [9, Theorem 2.2]. Hence,

$$
\left\|\left(T_{\lambda^{2}}^{2}\right)^{*} \overline{\partial^{j}} g\right\|_{2, D}=0
$$

Now we claim that

$$
\left\|\left(T_{\lambda}\right)^{*} \overline{\partial^{j}} g\right\|_{2, D}=0
$$

Indeed, since $T_{\lambda}$ is invertible for $\lambda \in D \backslash \sigma(T)$, (3.16) implies that

$$
\left\|\overline{\partial^{j}} g\right\|_{2, D \backslash \sigma(T)}=0
$$

Therefore

$$
\left\|\left(T_{\curlywedge}\right)^{*} \overline{\partial^{j}} g\right\|_{2, D \backslash \sigma(T)}=0 .
$$

Since $\sigma(T) \cap(-\sigma(T))=\emptyset$ and $\sigma\left(T^{*}\right)=\sigma(T)^{*},\left(T_{-\lambda}\right)^{*}$ is invertible for $\mathcal{\lambda} \in \sigma(T)$, therefore; from (3.17) we have

$$
\left\|\left(T_{\curlywedge}\right)^{*} \bar{\partial}^{j} g\right\|_{2, \sigma(T)}=0
$$

It is clear form (3.20); and (3.21) that

$$
\left\|\left(T_{\lambda}\right)^{*} \overline{\partial^{j}} g\right\|_{2, D}=0, \quad \text { for } j=0,1, \ldots, m .
$$

Thus Proposition 3.21 and (3.21) imply

$$
\left\|(I-P) \bar{\partial}^{j} g\right\|_{2, D}=0 \quad \text { for } j=0,1, \ldots, m-2,
$$

where $P$ denotes the orthogonal projection of $L^{2}(D, H)$ onto $A^{2}(D, H)$. 
Hence $\left(T_{\lambda^{2}}^{2}\right) P g=T_{\lambda^{2}}^{2} g=0$. Since $T^{2}$ has SVEP, $T$ has SVEP. Also $g=P g$ is analytic and $(T-\lambda) g(\lambda)=0$ for $\lambda \in D$. Hence $g=0$. Thus, $T_{\lambda}$ is one to one.

Corollary 3.23. If $T_{1}$ and $T_{2}$ are of class $[2 Q N]$ with $\sigma\left(T_{i}\right) \cap\left(-\sigma\left(T_{i}\right)\right)=\emptyset$, for $i=1,2$ and $T_{2} T_{1}=0$. Then

$$
\left(T_{1}+T_{2}\right)_{\curlywedge}: W_{2}^{m}(D, H) \longrightarrow W_{2}^{m}(D, H)
$$

is one to one.

Proof. If $f \in W_{2}^{m}(D, H)$ is such that $\left(T_{1}+T_{2}\right)_{\lambda} f=0$. Since $T_{2} T_{1}=0$, we get $\left(T_{2}-\lambda\right) T_{2} f=0$. Since $\left(T_{2}\right)_{\lambda}$ is one to one, $T_{2} f=0$. Hence, $\left(T_{1}\right)_{\lambda} f=0$. Since $\left(T_{1}\right)_{\lambda}$ is one to one, $f=0$.

The following corollary shows that the nilpotent perturbation of operators in [2QN] satisfying SVEP satisfies SVEP.

Corollary 3.24. If an operator $T \in \mathcal{L}(H)$ is a nilpotent perturbation of a 2-power quasi-normal operator $S$, that is, $T=S+N$, where $S$ is of class [2QN], $S$ and $N$ commute, and $N^{m}=0$. If $\sigma(S) \cap(-\sigma(S))=\emptyset$, then $T_{\curlywedge}$ is one-to-one.

Proof. If $g \in W_{2}^{m}(D, H)$ is such that $T_{\lambda} g=0$, then

$$
S_{\lambda} g=-N g
$$

Hence $S_{\lambda} N^{j-1} g=-N^{j} g$ for $j=1,2, \ldots, m$. We prove that $N^{j} g=0$ for $j=0,1, \ldots, m-$ 1 by indication. Since $N^{m}=0, S_{\lambda} N^{m-1} g=-N^{m} g=0$. Since $S_{\lambda}$ is one-to-one by Theorem $3.22 N^{m-1} g=0$. Assume it is true when $j=k$, that is, $N^{k} g=0$. From (3.25), we get

$$
S_{\curlywedge} N^{k-1} g=-N^{k} g=0 .
$$

Since $S_{\lambda}$ is one-to-one from Theorem 3.22, $N^{k-1} g=0$. By indication we have $g=0$. Hence $T_{\lambda}$ is one-to-one.

An operator $T \in \mathcal{L}(H)$ is said to be the following.

(1) It is left invertible if there is an operator $S \in \mathcal{L}(H)$ such that $S T=I$, where $I$ denotes the identity operator. The operator $S$ is called a left inverse of $T$.

(2) It is right invertible if there is an operator $R \in \mathcal{L}(X)$ such that $T R=I$. The operator $R$ is called a right inverse of $T$ (see [16]).

Corollary 3.25. If $T$ is of class [2QN] with the property $\sigma(T) \cap(-\sigma(T))=\emptyset$, and if $S$ is a left invertible operator with the left inverse $R$, then the operator $(S T R)_{\lambda}: W_{2}^{m}(D, H) \rightarrow W_{2}^{m}(D, H)$ is one-to-one.

Proof. If $g \in W_{2}^{m}(D, H)$ is such that $(\mathrm{STR}) \_g=0$, then

$$
T_{\lambda} R g=0
$$


Hence for $j=0,1, \ldots, m$ we have $T_{\lambda} R \bar{\partial}^{j} g=0$. From Theorem 3.22, we get $R \bar{\partial}^{j} g=0$ for $j=0,1, \ldots, m$.

Thus, $\operatorname{STR} \bar{\partial}^{j} g=0$ for $j=0,1, \ldots, m$. It follows that $\lambda \bar{\partial}^{j} g=0$ for $j=0,1, \ldots, m$. By application of [7, Proposition 2.1] with $T=(0)$, we have

$$
\|(I-P) g\|_{2, D}=0,
$$

where $P$ denotes the orthogonal projection of $L^{2}(D, H)$ onto the Bergman space $A^{2}(D, H)$. Hence $\lambda g=\lambda P g=0$. From [17, Corollary 10.7], there exists a constant $c>0$ such that

$$
c\|P g\|_{2, D} \leq\|\lambda P g\|_{2, D} .
$$

So $g=P g=0$. Thus, $(\mathrm{STR})_{\mathcal{\lambda}}$ is one-to-one.

Corollary 3.26. If $T$ is of class [2QN] with the property $\sigma(T) \cap(-\sigma(T))=\emptyset$, and if $S$ is a right invertible operator with the right inverse $R$ then the operator $(R T S)_{\lambda}: W_{2}^{m}(D, H) \rightarrow W_{2}^{m}(D, H)$, is one-to-one.

Now we are ready to prove our main theorem.

Theorem 3.27. If $T$ is of class $[2 Q N]$ with the property that $\sigma(T) \cap(-\sigma(T))=\emptyset$ and let $D$ be a bounded disk which contains $\sigma(T)$, then the operator $V: H \rightarrow H(D)$, defined by

$$
V g=1 \otimes g+\overline{T_{\lambda} W_{2}^{m}(D, H)}=[\widehat{1 \otimes g}]
$$

is one to one and has closed range, where $H(D)$ is as in (2.11).

Proof. First, we will prove that if $\left\{g_{k}\right\}_{1}^{\infty} \subset H$ and $\left\{f_{k}\right\}_{1}^{\infty} \subset W_{2}^{m}(D, H)$ are sequences such that

$$
\lim _{k \rightarrow \infty}\left\|1 \otimes g_{k}+T_{\lambda} f_{k}\right\|_{W_{2}^{m}}=0,
$$

then $\lim _{k \rightarrow \infty} g_{k}=0$.

By the definition of the norm of a Sobolev space, (3.31) implies that

$$
\lim _{k \rightarrow \infty}\left\|T_{\lambda} \bar{\partial}^{j} f_{k}\right\|_{2, D}=0 \quad \text { for } j=1, \ldots, m \text {. }
$$

From (3.32) we get

$$
\lim _{k \rightarrow \infty}\left\|T_{\lambda^{2}}^{2} \bar{\partial}^{j} f_{k}\right\|_{2, D}=0 \quad \text { for } j=1, \ldots, m \text {. }
$$


International Journal of Mathematics and Mathematical Sciences

Since $T^{2}$ is normal,

$$
\lim _{k \rightarrow \infty}\left\|\left(T_{\lambda^{2}}^{2}\right)^{*} \bar{\partial}^{j} f_{k}\right\|_{2, D}=0 \quad \text { for } j=1, \ldots, m
$$

Since $T_{\lambda}$ is invertible for $\lambda \in D \backslash \sigma(T)$, (3.32) implies that

$$
\lim _{k \rightarrow \infty}\left\|\bar{\partial}^{j} f_{k}\right\|_{2, D \backslash \sigma(T)}=0
$$

Therefore

$$
\lim _{k \rightarrow \infty}\left\|\left(T_{\curlywedge}\right)^{*} \bar{\partial}^{j} f_{k}\right\|_{2, D \backslash \sigma(T)}=0 \quad \text { for } j=1, \ldots, m
$$

Since $\sigma(T) \cap(-\sigma(\mathrm{T}))=\emptyset$ and $\sigma\left(T^{*}\right)=\sigma(T)^{*}$, it is clear that $\left(T_{-\lambda}\right)^{*}$ is invertible for $\lambda \in \sigma(T)$. Therefore from (3.34), we have

$$
\lim _{k \rightarrow \infty}\left\|\left(T_{\lambda}\right)^{*} \bar{\partial}^{j} f_{k}\right\|_{2, \sigma(T)}=0
$$

Hence, from (3.36) and (3.37) we get

$$
\lim _{k \rightarrow \infty}\left\|\left(T_{\lambda}\right)^{*} \bar{\partial}^{j} f_{k}\right\|_{2, D}=0, \quad j=1, \ldots, m .
$$

Then by Proposition 3.21, we have

$$
\lim _{k \rightarrow \infty}\left\|(I-P) \bar{\partial}^{j} f_{k}\right\|_{2, D}=0, \quad j=1, \ldots, m-2 .
$$

By (3.31) and (3.39), we have

$$
\lim _{k \rightarrow \infty}\left\|1 \otimes g_{k}+T_{\lambda} P f_{k}\right\|_{2, D}=0
$$

Let $\Gamma$ be a curve in $D$ surrounding $\sigma(T)$. Then

$$
\lim _{k \rightarrow \infty}\left\|P f_{k}+\left(T_{\lambda}\right)^{-1}\left(1 \otimes g_{k}\right)\right\|=0
$$

uniformly for $\lambda \in \Gamma$ by (3.40). Hence by Riesz-Dunford functional calculus

$$
\lim _{k \rightarrow \infty}\left\|\frac{1}{2 \pi i} \int_{\Gamma} P f_{k}(z) d z+g_{k}\right\|=0 .
$$


But by Cauchy's theorem

$$
\int_{\Gamma} P f_{k}(z) d z=0
$$

Hence $\lim _{k \rightarrow \infty} g_{k}=0$. Thus the map $V$ is one-to-one and has closed range.

Corollary 3.28. If $T$ is of class $[2 Q N]$ with the property that $\sigma(T) \cap(-\sigma(T))=\emptyset$, then $T$ is subscalar of order $m \geq 2$.

Proof. Consider an arbitrary bounded open disk $D$ in $\mathbb{C}$ that contains $\sigma(T)$ and the quotient space given in (2.11).

Let $M_{\curlywedge}$ be the multiplication operator by $\lambda$ on $W_{2}^{m}(D, H)$. Then $M_{\lambda}$ is a scalar operator of order $m$, and its spectral distribution is

$$
\Phi_{M}: C_{0}^{m}(\mathbb{C}) \longrightarrow \mathcal{L}\left(W_{2}^{m}(D ; H)\right), \quad \Phi_{M}(f)=M_{f},
$$

where $M_{f}$ is the multiplication operator by $f \in C_{0}^{m}(\mathbb{C})$. Let $S \equiv \widehat{M_{\lambda}}$. Since $T_{\lambda} W_{2}^{m}(D ; H)$ is invariant under every operator $M_{f}$, we infer that $S$ is a scalar operator of order $m$ with spectral distribution $\widehat{\Phi}$.

Let $V$ be the operator

$$
V g=1 \otimes g+\overline{T_{\curlywedge} W_{2}^{m}(D, H)}
$$

from $H$ into $H(D)$. Then we have the following commutative diagram

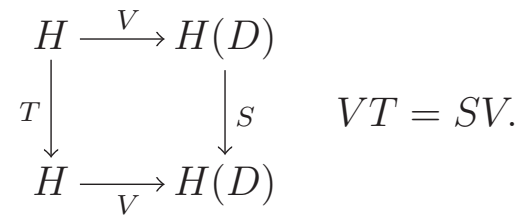

By the previous theorem the operator $V$ is a topological isomorphism of $H$ into $R(V)$. The relation $V T=S V$ shows that $R(V)$ is $S$-invariant. Hence $S$ is an extension of the operator $V$, so this operator is subscalar. Since $V$ is invertible on $R(V)$, then the operator $T$ is subscalar of order $m$. On the other hand from [18, Theorem 4.3] we deduce that $m \geq 2$ and the theorem is proved.

Corollary 3.29. If $T$ is of class $[2 Q N]$ with the property that $\sigma(T) \cap(-\sigma(T))=\emptyset$, then $T$ has Bishop's property $(\beta)$.

Proof. It follows from Corollary 3.28 and [18, Lemma 2.1].

In [19] the authors study some operators with the single-valued extension property. In the following propositions we extend some of these results to operators with the Bishop's property $(\beta)$. 
Proposition 3.30. Let $T \in \mathcal{L}\left(\oplus_{i=1}^{k} H\right)$ be the following $k \times k$ triangular operator matrix

$$
T=\left(\begin{array}{cccccc}
T_{11} & T_{12} & T_{13} & \ldots & \ldots & T_{1 k} \\
0 & T_{22} & T_{23} & \ldots & \ldots & T_{2 k} \\
0 & 0 & T_{33} & \ldots & \ldots & T_{3 k} \\
0 & 0 & 0 & \ldots & \ldots & \ldots \\
0 & \ldots & \ldots & \ldots & \ldots & T_{k k}
\end{array}\right)
$$

Assume that $T_{i i}$ is of class $[2 Q N]$ and satisfies $\sigma\left(T_{i i}\right) \cap\left(-\sigma\left(T_{i i}\right)\right)=\emptyset$ for $i=1,2, \ldots, k-1$ and $T_{k k}$ is nilpotent. Then $T$ has Bishop's property $(\beta)$.

Proof. Let $f_{p}=\oplus_{i=1}^{k} f_{p}^{i}: D \rightarrow \oplus_{i=1}^{k} H$ be a sequence of analytic functions such that $T_{\lambda} f_{p}(\lambda) \rightarrow 0$ uniformly on every compact subset $K$ of an open set $D$ of $\mathbb{C}$; then we have

$$
\begin{gathered}
\left(T_{11}\right)_{\lambda} f_{p}^{1}(\lambda)+T_{12} f_{p}^{2}(\lambda)+T_{13} f_{p}^{3}(\lambda) \cdots+T_{1 k} f_{p}^{k}(\lambda) \longrightarrow 0, \\
\left(T_{22}\right)_{\lambda} f_{p}^{2}(\lambda)+T_{23} f_{p}^{3}(\lambda)+\cdots+T_{2 k} f_{p}^{k} \longrightarrow 0, \\
\left(T_{33}\right)_{\lambda} f_{p}^{3}(\lambda)+\cdots+T_{3 k} f_{p}^{k}(\lambda) \longrightarrow 0, \\
\vdots \quad \\
\vdots \quad \\
\left(T_{k-1 k-1}\right)_{\lambda} f_{p}^{k-1}(\lambda)+T_{k-1 k} f_{p}^{k}(\lambda) \longrightarrow 0, \\
\left(T_{k k}\right)_{\lambda} f_{p}^{k}(\lambda) \longrightarrow 0 .
\end{gathered}
$$

Since $T_{k k}^{m}=0, \lambda T_{k k}^{m-1} f_{p}^{k}(\lambda) \rightarrow 0$ and hence $T_{k k}^{m-1} f_{p}^{k}(\lambda) \rightarrow 0$ if $\lambda \neq 0$. Since $\left(T_{k k}\right)_{\lambda} f_{p}^{k}(\lambda) \rightarrow 0$ from $(3.48) \lambda T_{k k}^{m-2} f_{p}^{k}(\lambda) \rightarrow 0$. By the same reason, $T_{k k}^{m-3} f_{p}^{k}(\lambda) \rightarrow 0$. By repeating this procedure, we finally achieve

$$
f_{p}^{k}(\lambda) \longrightarrow 0
$$

uniformly on $K$. Then we obtain the following equation: $\left(T_{k-1 k-1}\right)_{\lambda} f_{p}^{k-1} \rightarrow 0$ uniformly on every compact $K$. Since $T_{k-1 k-1}$ has Bishop's property $(\beta)$ from Corollary $3.29, f_{p}^{k-1}(\lambda) \rightarrow 0$ uniformly on $K$. By repeating this process we prove that $f_{p}^{1}(\lambda) \rightarrow 0$ uniformly on $K$.

Hence $\left\{f_{p}=f_{p}^{1} \oplus f_{p}^{2} \oplus \cdots \oplus f_{p}^{k}\right\}$ converge uniformly to 0 on any compact subset $K$ of $D$, and so $T$ has the Bishop's property $(\beta)$.

Proposition 3.31. Let $T$ be as in Proposition 3.30. Then if $T_{i i}$ has Bishop's property $(\beta)$ for $i=$ $1, \ldots, k$, then $T$ has Bishop's property $(\beta)$.

Proof. The proof is identical to the proof of Proposition 3.30. 
Proposition 3.32. Let $T \in \mathcal{L}\left(\oplus_{i=1}^{k} H\right)$ be the following $k \times k$ triangular operator matrix:

$$
T=\left(\begin{array}{cccccc}
T_{11} & T_{12} & T_{13} & \ldots & \ldots & T_{1 k} \\
0 & T_{22} & T_{23} & \ldots & \ldots & T_{2 k} \\
0 & 0 & T_{33} & \ldots & \ldots & T_{3 k} \\
0 & 0 & 0 & \ldots & \ldots & \ldots \\
0 & \ldots & \ldots & \ldots & \ldots & T_{k k}
\end{array}\right)
$$

Assume that $T$ is of class [2QN] and $\sigma(T) \cap(-\sigma(T))=\emptyset$. If $T_{11} T_{i j}=T_{i j} T_{j j}$ for $i=1, \ldots j$ and $j=1,2, \ldots, k$, then $T_{j j}$ has Bishop's property $(\beta)$ for $j=1,2, \ldots, k$.

Proof. Let $f_{p}^{j}: D \rightarrow H$ be a sequence of analytic functions such that $\left(T_{j j}\right)_{\lambda} f_{p}^{j} \rightarrow 0$ uniformly on every compact subset $K$ of $D$, then we have for $j=1,2, \ldots, k$

$$
\begin{aligned}
T_{\lambda}\left(T_{i j} f_{p}^{j}(\lambda) \oplus \cdots \oplus 0\right) & =\left(T_{11}\right)_{\lambda}\left(T_{i j} f_{p}^{j}(\lambda)\right) \oplus 0 \oplus \cdots \oplus 0 \\
& =T_{i j}\left(T_{j j}\right)_{\lambda} f_{p}^{j}(\lambda) \oplus \cdots \oplus 0 \\
& \longrightarrow 0,
\end{aligned}
$$

for $i=1,2, \ldots, j$. Since $T$ has Bishop's property $(\beta)$, we get that $T_{i j} f_{p}^{j}(\lambda) \rightarrow 0$ uniformly on $K$ for $j=1,2, \ldots, k$. We have

$$
T_{\lambda}\left(f_{p}^{1} \oplus \cdots \oplus 0\right)=\left(T_{11}\right)_{\lambda} f_{p}^{1}(\lambda) \oplus \cdots \oplus 0 \longrightarrow 0
$$

and for $j=2,3, \ldots, k$,

$$
\begin{aligned}
T_{\lambda}\left(0 \oplus \cdots \oplus f_{p}^{j} \oplus \cdots \oplus 0\right) & =\left(-T_{1 j} f_{p}^{j}(\lambda)\right) \oplus \cdots \oplus\left(-T_{j-1 j} f_{p}^{j}(\lambda)\right) \oplus\left(T_{j j}\right)_{\lambda}\left(f_{p}^{j}(\lambda)\right) \oplus 0 \oplus \cdots \oplus 0 \\
& \longrightarrow 0
\end{aligned}
$$

Since $T$ has Bishop's property $(\beta), f_{p}^{j}(\lambda) \rightarrow 0$ uniformly on $K$ for $j=1,2, \ldots, k$. Thus, $T_{j j}$ has Bishop's property $(\beta)$.

Proposition 3.33. Let $T$ be as in Proposition 3.31. Then if $T$ has Bishop's property $(\beta)$ and $T_{11} T_{i j}=$ $T_{i j} T_{j j}$ for $j=1,2, \ldots, k$ and $j=1,2, \ldots, k$, then $T_{j j}$ has Bishop's property $(\beta)$ for $j=1,2, \ldots, k$.

Proof. The proof is identical to the proof of Proposition 3.32. 


\section{Berberian Extension}

Denote by $l^{\infty}(H)$ the space of all sequences $\left(x_{n}\right)_{n}$, with $x_{n} \in H, n=1,2,3, \ldots$ such that $\left\|x_{n}\right\|$ is bounded. Let $c_{0}(H)$ denote the subspace of all null sequences of $H$ (those such that $\left\|x_{n}\right\| \rightarrow$ $0)$. If we set $\left\|\left(x_{n}\right)\right\|=\sup \left\|x_{n}\right\|$ for every sequence $\left(x_{n}\right)$, this defines a seminorm on $l^{\infty}(H)$, which is zero exactly on the elements of $c_{0}(H)$. By means of the space $l^{\infty}(H)$ and the Banach limits, Berberian [20] constructed an extension $H^{\circ}$ of $H$ and obtained a homomorphism form operators $T \in \mathcal{L}(H)$ to operators $T^{\circ} \in \mathcal{L}\left(H^{\circ}\right)$ such that $T^{\circ}$ is an extension of $T$.

Theorem 4.1 (Berberian extention [20]). Let $H$ be a complex Hilbert space. Then there exists a Hilbert space $H^{\circ} \supset H$ and a map

$$
\Phi: \mathcal{L}(H) \longrightarrow \mathcal{L}\left(H^{\circ}\right): T \longmapsto T^{\circ},
$$

satisfying: $\Phi$ which is an ${ }^{*}$-isometric isomorphism preserving the order such that

(1) $\left(T^{*}\right)^{\circ}=\left(T^{\circ}\right)^{*}$,

(2) $(\lambda T+\mu S)^{\circ}=\lambda T^{\circ}+\mu S^{0}$,

(3) $\left(I_{H}\right)^{\circ}=I_{H^{\circ}}$,

(4) $(T S)^{\circ}=T^{\circ} S^{\circ}$,

(5) $\left\|T^{\circ}\right\|=\|T\|$,

(6) $T^{\circ} \leq S^{\circ}$ if $T \leq S$,

(7) $\sigma\left(T^{\circ}\right)=\sigma(T), \sigma_{a p}(T)=\sigma_{a p}\left(T^{\circ}\right)=\sigma_{p}\left(T^{\circ}\right)$,

(8) if $T$ is a positive operator, then $\left(T^{\alpha}\right)^{\circ}=\left|T^{\circ}\right|^{\alpha}$ for all $\alpha>0$.

An operator is said to be reducible if it has a nontrivial reducing subspace. If an operator is not reducible, then it is called irreducible.

Proposition 4.2 (see [21]). If $T$ is an irreducible operator, then $T^{\circ}$ is an irreducible operator.

Lemma 4.3. Let $D$ be a subset of $\mathbb{C}, z_{0}, R>0$, such that $\overline{B\left(z_{0}, R\right)}=\left\{z \in \mathbb{C} ;\left|z-z_{0}\right| \leq R\right\} \subset$ $D$, let $g_{n}: D \rightarrow H$ be a sequence of analytic functions, and let the Taylor expansion of $g_{n}$ be

$$
g_{n}(z)=\sum_{k=0}^{\infty} a_{n k}\left(z-z_{0}\right)^{k}, \quad\left|z-z_{0}\right|<R
$$

If $g_{n}$ is uniformly bounded on $\overline{B\left(z_{0}, R\right)}$ (i.e., $\left.M=\sup _{n \geq 1}\left\|g_{n}(z)\right\|_{\overline{B\left(z_{0}, R\right)}}<\infty\right)$, then

$$
\left\|g_{n}(z)-g_{n}\left(z_{0}\right)\right\| \leq \frac{M r}{R-r}, \quad z \in \overline{B\left(z_{0}, r\right)}, 0<r<R
$$


Proof. For all $n$ and $z \in \overline{B\left(z_{0}, r\right)}$ with $0<r<R$, by Cauchy's integral formula, we get the following inequality:

$$
\begin{aligned}
\left\|g_{n}(z)-g_{n}\left(z_{0}\right)\right\| & =\left\|\frac{1}{2 i \pi} \int_{\left|u-z_{0}\right|=R} \frac{g_{n}(u)}{u-z} d u-\frac{1}{2 i \pi} \int_{\left|u-z_{0}\right|=R} \frac{g_{n}(u)}{u-z_{0}} d u\right\| \\
& \leq \frac{1}{2 \pi} \int_{\left|u-z_{0}\right|=R} \frac{\left|z-z_{0}\right|\left\|g_{n}(u)\right\|}{|u-z|\left|u-z_{0}\right|}|d u| \\
& \leq \frac{M r}{R-r} .
\end{aligned}
$$

Remark 4.4. Let $D$ be an open subset of $\mathbb{C}$. A sequence of analytic functions $g_{\mathrm{n}}: D \rightarrow H$ converges uniformly to 0 on every compact subset $K$ of $D$ if and only if for any $\epsilon>0$ and any $z_{0} \in D$ there exists $r>0$ and $n_{0} \in \mathrm{N}$ such that $\overline{B\left(z_{0} ; r\right)} \subset D$ and $\left\|g_{n}\right\|_{\overline{B\left(z_{0}, r\right)}}<\epsilon$ for all $n>n_{0}$.

\section{Single-valued Extension Property for $m$-Partial Isometries}

In this section we examine the properties of SVEP and Bishop's property $(\beta)$ for some $m$-partial isometries operators by using an approach which is different from that used in Section 3 . We recall the definition of an $m$-partial isometry given by (1.4) and the operator $B_{m}(T)$.

Definition 5.1 (see [8]). An operator $T \in \mathcal{L}(H)$ is called an $m$-isometry if

$$
B_{m}(T)=\sum_{k=0}^{m}(-1)^{k}\left(\begin{array}{l}
m \\
k
\end{array}\right) T^{* m-k} T^{m-k}=0 .
$$

Remark 5.2. It is easy to see that $T \in \mathcal{L}(H)$ is an $m$-partial isometry if and only if

$$
B_{m}(T) x=\sum_{k=0}^{m}(-1)^{k}\left(\begin{array}{l}
m \\
k
\end{array}\right) T^{* m-k} T^{m-k}(x)=0, \quad \forall x \in N(T)^{\perp},
$$

which shows that the class of $m$-partial isometries generalizes those of $m$-isometries and partial isometries.

Theorem 5.3 (see [10]). If $T \in \mathcal{L}(H)$ is reducible, that is, if it has a nontrivial reducing subspace $N(T)$, then the following properties are equivalent.

(1) $T$ is an m-partial isometry.

(2) $\left.T\right|_{N(T)^{\perp}}$ is an m-isometry.

Proposition 5.4 (see [10]). Let $T$ be a reducible m-partial isometry. Then

(1) $\lambda \in \sigma_{a p}(T) \backslash\{0\}$ implies $\bar{\lambda} \in \sigma_{a p}\left(T^{*}\right)$, that is, if $T_{\lambda} x_{n} \rightarrow 0$ for some sequence of bounded vectors $\left\{x_{n}\right\} \subset H$, then $\left(T_{\curlywedge}\right)^{*} x_{n} \rightarrow 0$, 
International Journal of Mathematics and Mathematical Sciences

(2) $\lambda \in \sigma_{p}(T) \backslash\{0\}$ implies $\bar{\lambda} \in \sigma_{p}\left(T^{*}\right)$,

(3) eigenvectors of $T$ corresponding to distinct eigenvalues are orthogonal, that is, $N\left(T_{\lambda}\right) \perp$ $N\left(T_{\mu}\right)$ if $\lambda, \mu \in \sigma_{p}(T), \lambda \neq \mu$.

Lemma 5.5. Let $T$ be a reducible m-partial isometry and let $\lambda, \mu \in \sigma_{a p}(T)$ and $\left(x_{n}\right),\left(y_{n}\right)$ be sequences of bounded vectors in $H$ such that $\lambda \neq \mu$ and

$$
\left\|T_{\lambda} x_{n}\right\| \longrightarrow 0, \quad\left\|T_{\mu} y_{n}\right\| \longrightarrow 0 \quad(\text { as } n \longrightarrow \infty)
$$

Then we have

$$
\left\langle x_{n} \mid y_{n}\right\rangle \longrightarrow 0 \quad(\text { as } n \longrightarrow \infty)
$$

Proof. We may assume that $\mu \neq 0$. Then from Proposition 5.4(1) we have $\left\|\left(T_{\mu}\right)^{*} y_{n}\right\| \rightarrow 0$ as $n \rightarrow \infty$. Hence,

$$
(\lambda-\mu)\left\langle x_{n} \mid y_{n}\right\rangle=-\left\langle T_{\lambda} x_{n} \mid y_{n}\right\rangle+\left\langle x_{n} \mid\left(T_{\mu}\right)^{*} y_{n}\right\rangle \longrightarrow 0, \quad n \longrightarrow \infty
$$

which implies (5.4) in view of $\lambda \neq \mu$ and the proof is complete.

Theorem 5.6. Any reducible m-partial isometry has SVEP.

Proof. Let $U$ be a bounded subset of $\mathbb{C}$ and let $f: U \rightarrow H$ be an analytic function such that

$$
T_{\lambda} f(\lambda)=0 \quad \text { for } \lambda \in U
$$

Since $N\left(T_{\lambda}\right) \perp N\left(T_{\mu}\right), \lambda \neq \mu$ (Proposition 5.4(3)), we have

$$
\|f(\lambda)\|^{2}=\lim _{\mu \rightarrow \lambda}\langle f(\lambda) \mid f(\mu)\rangle=0 .
$$

This shows that $f(\lambda)=0$.

Lemma 5.7. If $T$ is an m-partial isometry, then $T^{o}$ is also an m-partial isometry.

Proof. It is a consequence of the properties of $T^{o}$ (see Theorem 4.1).

Theorem 5.8. If $T$ is an m-partial isometry with a nontrivial reducing space $N(T)$, then $T^{o}$ has the single-valued extension property (SVEP).

Proof. To prove that $T^{\circ}$ has SVEP, let $\lambda \in \sigma_{a p}\left(T^{\circ}\right)-\{0\}$. Since $\sigma_{a p}\left(T^{\circ}\right)=\sigma_{a p}(T)$ by Theorem 4.1, $\bar{\lambda} \in \sigma_{a p}\left(T^{*}\right)=\sigma_{a p}\left(\left(T^{\circ}\right)^{*}\right)$. In particular if $\lambda \in \sigma_{p}\left(T^{\circ}\right)$, then $\bar{\lambda} \in \sigma_{p}\left(\left(T^{\circ}\right)^{*}\right)$. Hence, $N\left(\left(T_{\lambda}\right)^{\circ}\right) \perp$ $N\left(\left(T_{\mu}\right)^{\circ}\right)$ for $\lambda$ and $\mu \in \sigma_{p}\left(T^{\circ}\right)$ with $\lambda \neq \mu$. In a similar way as in the proof of Theorem 5.6, we can see that $T^{\circ}$ has SVEP. 


\section{Acknowledgment}

The author would like to express their gratitude to the referees for their helpful and many valuable suggestions.

\section{References}

[1] D. Xia, Spectral Theory of Hyponormal Operators, vol. 10 of Operator Theory: Advances and Applications, Birkhäuser, Basel, Switzerland, 1983.

[2] E. Ko, "Properties of a kth root of a hyponormal operator," Bulletin of the Korean Mathematical Society, vol. 40, no. 4, pp. 685-692, 2003.

[3] E. Ko, H.-W. Nam, and Y. Yang, "On totally k-paranormal operators," Czechoslovak Mathematical Journal, vol. 56(131), no. 4, pp. 1265-1280, 2006.

[4] E. Ko, "k-quasihyponormal operators are subscalar," Integral Equations and Operator Theory, vol. 28, no. 4, pp. 492-499, 1997.

[5] H. C. Rhaly, Jr., "Posinormal operators," Journal of the Mathematical Society of Japan, vol. 46, no. 4, pp. 587-605, 1994.

[6] K. Tanahashi, "On log-hyponormal operators," Integral Equations and Operator Theory, vol. 34, no. 3, pp. 364-372, 1999.

[7] M. Putinar, "Hyponormal operators are subscalar," Journal of Operator Theory, vol. 12, no. 2, pp. 385395, 1984.

[8] J. Agler and M. Stankus, "m-isometric transformations of Hilbert space. I," Integral Equations and Operator Theory, vol. 21, no. 4, pp. 383-429, 1995.

[9] O. A. M. Sid Ahmed, "On the class of $n$-power quasi-normal operators on Hilbert space," Bulletin of Mathematical Analysis and Applications, vol. 3, no. 2, pp. 213-228, 2011.

[10] A. Saddi and O. A. M. Sid Ahmed, "m-partial isometries on Hilbert spaces," International Journal of Functional Analysis, vol. 2, no. 1, pp. 67-83, 2010.

[11] K. B. Laursen and M. M. Neumann, An Introduction to Local Spectral Theory, vol. 20 of London Mathematical Society Monographs New Series, The Clarendon Press Oxford University Press, Oxford, UK, 2000.

[12] P. R. Halmos, A Hilbert Space Problem Book, vol. 19 of Graduate Texts in Mathematics, Springer, New York, NY, USA, 2nd edition, 1982, Encyclopedia of Mathematics and its Applications 17.

[13] C. Kitai, Invariant closed sets for linear operators, Ph.D. thesis, University of Toronto, Toronto, Canada, 1982.

[14] D. A. Herrero and C. Kitai, "On invertible hypercyclic operators," Proceedings of the American Mathematical Society, vol. 116, no. 3, pp. 873-875, 1992.

[15] S. I. Ansari, "Hypercyclic and cyclic vectors," Journal of Functional Analysis, vol. 128, no. 2, pp. 374-383, 1995.

[16] O. A. M. Sid Ahmed, "Some properties of m-isometries and minvertible on Banach spaces," Acta Mathematica Scientia, vol. 32, no. 2, pp. 520-530, 2012.

[17] J. B. Conway, Subnormal Operators, vol. 51 of Research Notes in Mathematics, Pitman (Advanced Publishing Program), Boston, Mass, USA, 1981.

[18] M.-K. Kim and E. Ko, "Square roots of hyponormal operators," Glasgow Mathematical Journal, vol. 41, no. 3, pp. 463-470, 1999.

[19] Y. Kim, E. Ko, and J. E. Lee, "Operators with the single valued extension property," Bulletin of the Korean Mathematical Society, vol. 43, no. 3, pp. 509-517, 2006.

[20] S. K. Berberian, "Approximate proper vectors," Proceedings of the American Mathematical Society, vol. 13, pp. 111-114, 1962.

[21] H. K. Cha, "On the semi-hyponormal operators on a Hilbert space," Korean Mathematical Society, vol. 12, no. 3, pp. 597-602, 1997. 


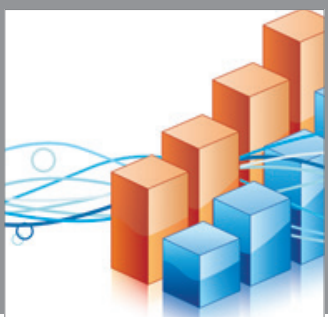

Advances in

Operations Research

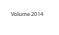

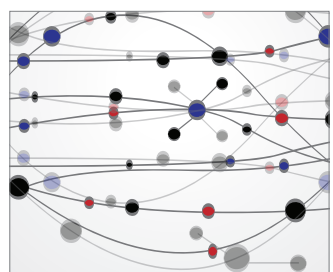

\section{The Scientific} World Journal
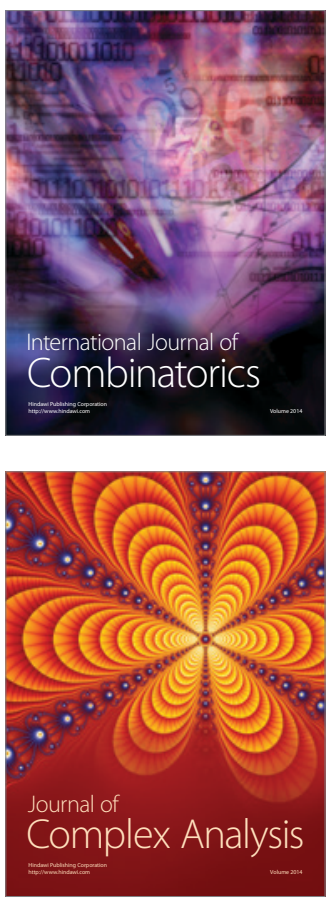

International Journal of

Mathematics and

Mathematical

Sciences
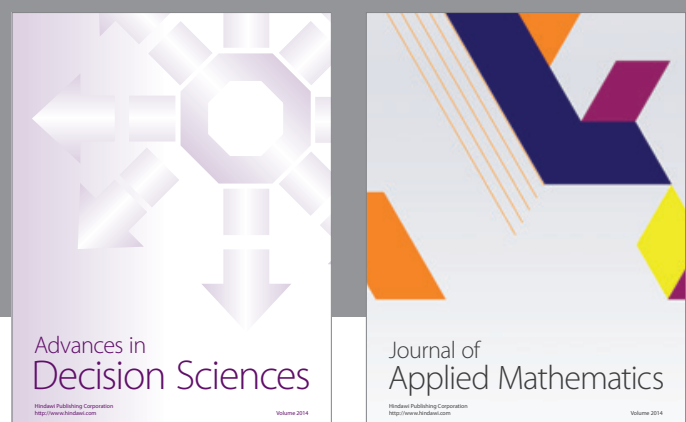

Journal of

Applied Mathematics
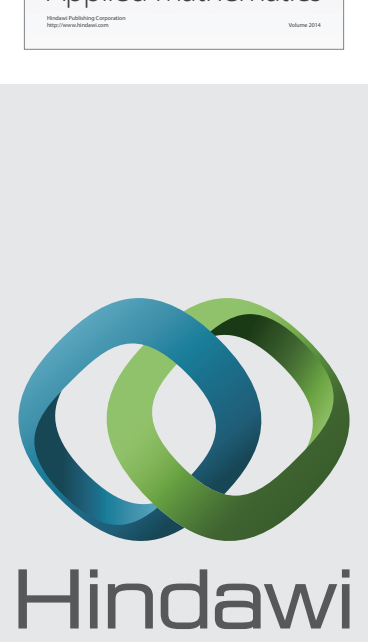

Submit your manuscripts at http://www.hindawi.com
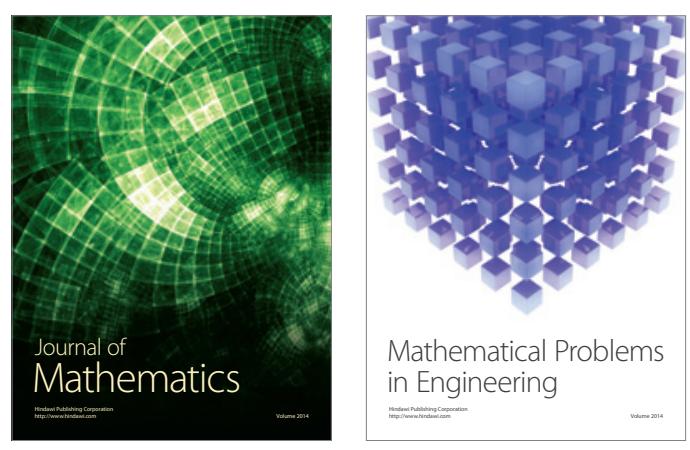

Mathematical Problems in Engineering
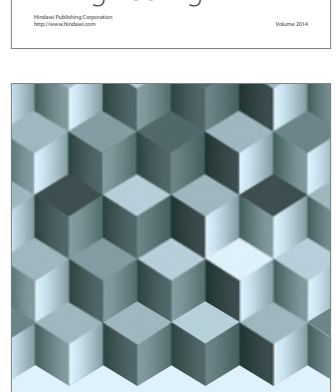

Journal of

Function Spaces
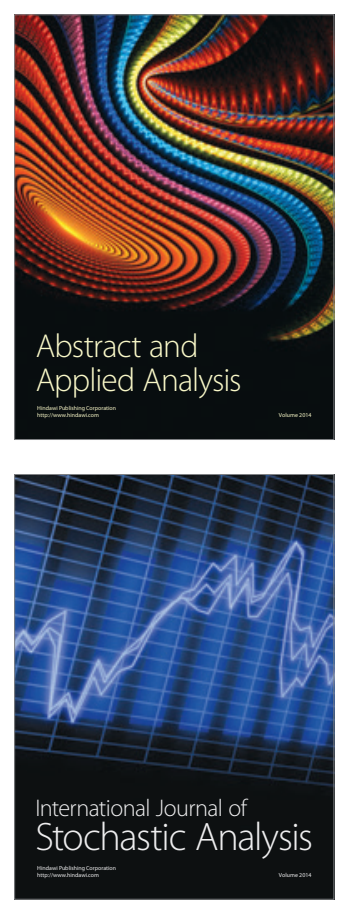

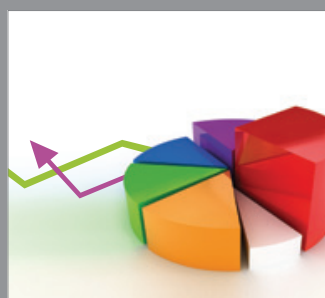

ournal of

Probability and Statistics

Promensencen
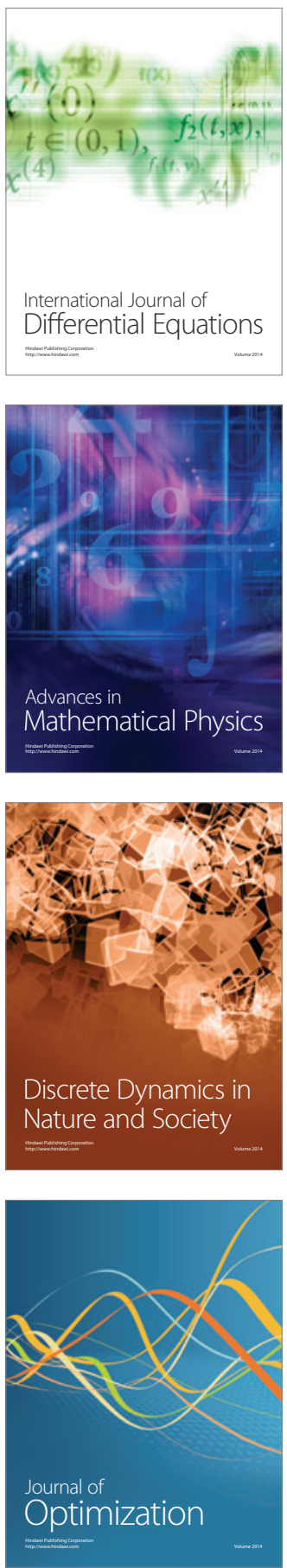